\title{
PROPERTIES OF A GENERALIZED UNIVERSAL COVERING SPACE OVER A DIGITAL WEDGE
}

\author{
SANG-EON HAN
}

\begin{abstract}
The paper studies an existence problem of a (generalized) universal covering space over a digital wedge with a compatible adjacency. In algebraic topology it is well-known that a connected, locally path connected, semilocally simply connected space has a universal covering space. Unlike this property, in digital covering theory we need to investigate its digital version which remains open.
\end{abstract}

\section{Introduction}

Useful tools from algebraic topology for studying digital topological properties of a (binary) digital space include a digital covering space. This has been studied in many papers including $[2,4,5,7,8,9,10,11$, $12,13,14,15,16,17,18]$. Motivated by the study of a covering space over a figure eight in algebraic topology [28], the recent papers [6] (see also $[3,11,16,17,18,19]$ ) studied its digital version, which plays an important role in classifying digital spaces. In algebraic topology, it is also well known that a universal covering space over the figure eight is an infinite tree with a fractal structure. But such a kind of approach cannot be available in digital covering theory. Indeed, we can find some intrinsic features of an infinite fold covering space over a digital wedge consisting of two simple closed $k$-curves. By using intrinsic features of a digital covering of a digital wedge, the papers $[16,18,19]$ study the generalized universal property which is the digital version of a universal covering space in algebraic topology. As shown in [16, 18, 19], compared with the algebraic topological version, the generalized universal property in digital covering theory has own properties.

Received July 6, 2010. Accepted August 7, 2010.

2000 MSC: 55Q70,55Q20,68R10,68U05.

Keywords: digital isomorphism, digital covering, simply $k$-connected, universal covering property, generalized universal covering space, compatible adjacency of a digital wedge. 
In the study of a digital wedge consisting of two simple closed $k$ curves, since we have mainly studied digital wedges consisting of two simple closed $k$-curves in $\mathbf{Z}^{2}, k \in\{4,8\}$, the other cases remain unsolved. Thus we need to expand the knowledge of a covering space over a digital wedge with a compatible adjacency.

This paper is organized as follows. Section 2 provides some basic notions and terminology. Section 3 reviews properties of a covering space over a digital wedge consisting of two simple closed $k$-curves with a compatible adjacency. Section 4 investigates properties of a generalized universal covering space over a digital wedge. Section 5 studies an existence problem of a generalized universal covering over a digital wedge.

\section{Preliminaries}

Let $\mathbf{Z}^{n}$ and $\mathbf{N}$ denote the sets of points in the Euclidean $n$ D space with integer coordinates and the set of natural numbers $n \in \mathbf{N}$, respectively. Since a digital image in $\mathbf{Z}^{n}$ can be regarded as a set with one of the $k$-adjacency relations of $\mathbf{Z}^{n}$ or a digital $k$-graph [27], in this paper we use the terminology digital space instead of digital image.

As a generalization of the $k$-adjacency relations of $2 \mathrm{D}$ and $3 \mathrm{D}$ digital space in $[25,27]$, we have used $k$-adjacency relations of $\mathbf{Z}^{n}$ for studying a multi-dimensional digital space $X \subset \mathbf{Z}^{n}$ induced from the following criterion $[4]$ (see also $[6,9]$ ):

For a natural number $m$ with $1 \leq m \leq n$, two distinct points $p=$ $\left(p_{i}\right)_{i \in[1, n]_{\mathbf{Z}}}$ and $q=\left(q_{i}\right)_{i \in[1, n]_{\mathbf{Z}}}$ are $k_{m^{-}}$(or $k(m, n)$-)adjacent if

- there are at most $m$ indices $i$ such that $\left|p_{i}-q_{i}\right|=1$ and

- for all other indices $i$ such that $\left|p_{i}-q_{i}\right| \neq 1, p_{i}=q_{i}$.

By using this operator, we established the following $k:=k_{m}:=$ $k(m, n)$-adjacency relations of $\mathbf{Z}^{n}$.

Proposition 2.1. [15] In $\mathbf{Z}^{n}$ we obtain the following $k$-adjacency relations.

$k:=k(m, n)=\sum_{i=n-m}^{n-1} 2^{n-i} C_{i}^{n}$, where $C_{i}^{n}=\frac{n !}{(n-i) ! i !}$.

We say that two subsets $(A, k)$ and $(B, k)$ of $(X, k)$ are $k$-adjacent to each other if $A \cap B=\emptyset$ and there are points $a \in A$ and $b \in B$ such that $a$ and $b$ are $k$-adjacent to each other [25]. We say that a set $X \subset \mathbf{Z}^{n}$ is $k$-connected if it is not a union of two disjoint non-empty sets that are not $k$-adjacent to each other [25]. For an adjacency relation $k$ of $\mathbf{Z}^{n}$, a simple $k$-path with $l+1$ elements in $\mathbf{Z}^{n}$ is assumed to be an injective 
sequence $\left(x_{i}\right)_{i \in[0, l]_{\mathbf{Z}}} \subset \mathbf{Z}^{n}$ such that $x_{i}$ and $x_{j}$ are $k$-adjacent if and only if either $j=i+1$ or $i=j+1$ [25]. If $x_{0}=x$ and $x_{l}=y$, then we say that the length of the simple $k$-path, denoted by $l_{k}(x, y)$, is the number $l$. A simple closed $k$-curve with $l$ elements in $\mathbf{Z}^{n}$, denoted by $S C_{k}^{n, l}$ [10], is the simple $k$-path $\left(x_{i}\right)_{i \in[0, l-1]_{\mathbf{Z}}}$, where $x_{i}$ and $x_{j}$ are $k$-adjacent if and only if $j=i+1(\bmod l)$ or $i=j+1(\bmod l)[25]$.

In order to study both digital continuity and various properties of a digital $k$-surface $[9,10]$, we have used the following digital $k$-neighborhood.

Definition 1. [6] For a digital space $(X, k)$ in $\mathbf{Z}^{n}$, the digital $k$ neighborhood of $x_{0} \in X$ with radius $\varepsilon$ is defined in $X$ to be the following subset of $X$

$$
N_{k}\left(x_{0}, \varepsilon\right)=\left\{x \in X \mid l_{k}\left(x_{0}, x\right) \leq \varepsilon\right\} \cup\left\{x_{0}\right\},
$$

where $l_{k}\left(x_{0}, x\right)$ is the length of a shortest simple $k$-path from $x_{0}$ to $x$ and $\varepsilon \in \mathbf{N}$.

Motivated by both the digital continuity in [27] and the $\left(k_{0}, k_{1}\right)$ continuity in [2] (see also [6]), the following notion of digital continuity has been often used for the study of multi-dimensional digital spaces.

Proposition 2.2. [13] Let $\left(X, k_{0}\right)$ and $\left(Y, k_{1}\right)$ be digital spaces in $\mathbf{Z}^{n_{0}}$ and $\mathbf{Z}^{n_{1}}$, respectively. A function $f: X \rightarrow Y$ is $\left(k_{0}, k_{1}\right)$-continuous if and only if for every $x \in X f\left(N_{k_{0}}(x, 1)\right) \subset N_{k_{1}}(f(x), 1)$.

Since a digital space can be considered to be a digital $k$-graph, we can use a $\left(k_{0}, k_{1}\right)$-isomorphism instead of a $\left(k_{0}, k_{1}\right)$-homeomorphism in $[2]$, as follows.

Definition 2. [7] For two digital spaces $\left(X, k_{0}\right)$ in $\mathbf{Z}^{n_{0}}$ and $\left(Y, k_{1}\right)$ in $\mathbf{Z}^{n_{1}}$, a map $h: X \rightarrow Y$ is called a $\left(k_{0}, k_{1}\right)$-isomorphism if $h$ is a $\left(k_{0}, k_{1}\right)$ continuous bijection and further, $h^{-1}: Y \rightarrow X$ is $\left(k_{1}, k_{0}\right)$-continuous. Then we use the notation $X \approx_{\left(k_{0}, k_{1}\right)} Y$. If $n_{0}=n_{1}$ and $k_{0}=k_{1}$, then we call it a $k_{0}$-isomorphism and use the notation $X \approx_{k_{0}} Y$.

For a digital space $(X, k)$ and $A \subset X,(X, A)$ is called a digital space pair with $k$-adjacency [9]. Furthermore, if $A$ is a singleton set $\left\{x_{0}\right\}$, then $\left(X, x_{0}\right)$ is called a pointed digital space [25]. Motivated by the pointed digital homotopy in [2], the following notion of relative digital homotopy to a subset $A \subset X$ is often used for studying a digital space $(X, k)$ in $\mathbf{Z}^{n}$ in terms of the $k$-homotopic thinning and the strong $k$-deformation retract in $[9,10]$ (see also [15]). If the identity map $1_{X}$ is $(k, k)$-homotopic relative to $\left\{x_{0}\right\}$ in $X$ to a constant map with space consisting of some $x_{0} \in X$, then we say that $\left(X, x_{0}\right)$ is pointed $k$-contractible [2]. Indeed, 
the notion of $k$-contractility is slightly different from the contractility in Euclidean topology [2] (see also [12]).

Unlike the two digital fundamental groups [1, 24], motivated by Khalimsky's digital $k$-fundamental group in [23], for a digital space $\left(X, x_{0}\right)$ the paper [2] establishes the digital $k$-fundamental group $\pi^{k}\left(X, x_{0}\right)$ which is a group [2], where the base point is assumed as a point which is not deletable by a strong deformation retract [11]. Besides, if $X$ is pointed $k$ contractible, then $\pi^{k}\left(X, x_{0}\right)$ is proved trivial [2]. Let $((X, A), k)$ be a digital space pair with $k$-adjacency. A map $f:\left((X, A), k_{0}\right) \rightarrow\left((Y, B), k_{1}\right)$ is called $\left(k_{0}, k_{1}\right)$-continuous if $f$ is $\left(k_{0}, k_{1}\right)$-continuous and $f(A) \subset B$ [8]. If $A=\{a\}, B=\{b\}$, we write $(X, A)=(X, a),(Y, B)=(Y, b)$, and we say that $f$ is a pointed $\left(k_{0}, k_{1}\right)$-continuous map [25]. Besides, a $\left(k_{0}, k_{1}\right)$-continuous map $f:\left(\left(X, x_{0}\right), k_{0}\right) \rightarrow\left(\left(Y, y_{0}\right), k_{1}\right)$ induces a group homomorphism $f_{*}: \pi^{k_{0}}\left(X, x_{0}\right) \rightarrow \pi^{k_{1}}\left(Y, y_{0}\right)$ given by $f_{*}([\alpha])=[f \circ \alpha]$, where $[\alpha] \in \pi^{k_{0}}\left(X, x_{0}\right)[2]$.

The following notion has been often used in digital $k$-homotopy theory and digital covering theory.

Definition 3. [6] A pointed $k$-connected digital space $\left(X, x_{0}\right)$ is called simply $k$-connected if $\pi^{k}\left(X, x_{0}\right)$ is a trivial group.

Theorem 2.3. [6] (see also [11]) $\pi^{k}\left(S C_{k}^{n, l}\right)$ is an infinite cyclic group. Precisely, $\pi^{k}\left(S C_{k}^{n, l}\right) \simeq(l \mathbf{Z},+)$, where $S C_{k}^{n, l}$ is not $k$-contractible and " $\simeq$ " means a group isomorphism.

\section{Generalized Universal Covering Space over a Digital Wedge}

Some properties of a digital covering space including the unique lifting property [6] and digital homotopy lifting theorem [5] have been substantially used in calculating $\pi^{k}\left(X, x_{0}\right)$ and classifying digital spaces $[9,10,11,12,13,14,15,16,17]$, proving an existence of a universal covering space [17] and studying the Cartesian product of universal covering property [16]. Let us now recall the axiom of a digital covering space which is equivalent to the earlier version in $[5,6]$. In this section we will refer to a simpler form of a digital covering space (see Proposition 3.2).

Proposition 3.1. [6] (see also [9]) Let $\left(E, k_{0}\right)$ and $\left(B, k_{1}\right)$ be digital spaces in $\mathbf{Z}^{n_{0}}$ and $\mathbf{Z}^{n_{1}}$, respectively. Let $p: E \rightarrow B$ be a $\left(k_{0}, k_{1}\right)$ continuous surjection. Suppose, for any $b \in B$, there exists $\varepsilon \in \mathbf{N}$ such that

(1) for some index set $M, p^{-1}\left(N_{k_{1}}(b, \varepsilon)\right)=\cup_{i \in M} N_{k_{0}}\left(e_{i}, \varepsilon\right)$ with $e_{i} \in$ 
$p^{-1}(b)$

(2) if $i, j \in M$ and $i \neq j$, then $N_{k_{0}}\left(e_{i}, \varepsilon\right) \cap N_{k_{0}}\left(e_{j}, \varepsilon\right)$ is an empty set; and

(3) the restriction map $p$ on $N_{k_{0}}\left(e_{i}, \varepsilon\right)$ is a $\left(k_{0}, k_{1}\right)$-isomorphism for all $i \in M$.

Then, the map $p$ is called a $\left(k_{0}, k_{1}\right)$-covering map and $(E, p, B)$ is said to be a $\left(k_{0}, k_{1}\right)$-covering. The digital space $E$ is called a $\left(k_{0}, k_{1}\right)$-covering space over $B$.

In Proposition 3.1 we may take $\varepsilon=1$ [9] (see also [3]). Recently, by using the surjection instead of the $\left(k_{0}, k_{1}\right)$-continuous surjection of Proposition 3.1, the paper [18] improves the axiom of a digital $\left(k_{0}, k_{1}\right)$ covering, as follows.

Proposition 3.2. [22] Let us replace the $\left(k_{0}, k_{1}\right)$-continuous surjection of Proposition 3.1 by a surjection. Then the map $p$ is a $\left(k_{0}, k_{1}\right)$ covering map.

Definition 4. [9] $A\left(k_{0}, k_{1}\right)$-covering $(E, p, B)$ is called a radius $n$ $\left(k_{0}, k_{1}\right)$-covering if $\varepsilon \geq n$.

According to Definition 4 , we clearly observe that a $\left(k_{0}, k_{1}\right)$-covering of Proposition 3.1 is obviously a radius $1-\left(k_{0}, k_{1}\right)$-covering $[9]$.

For three digital spaces $\left(E, k_{0}\right)$ in $\mathbf{Z}^{n_{0}},\left(B, k_{1}\right)$ in $\mathbf{Z}^{n_{1}}$, and $\left(X, k_{2}\right)$ in $\mathbf{Z}^{n_{2}}$, let $p: E \rightarrow B$ be a $\left(k_{0}, k_{1}\right)$-continuous map. For a $\left(k_{2}, k_{1}\right)$ continuous map $f:\left(X, k_{2}\right) \rightarrow\left(B, k_{1}\right)$, as the digital analogue of the lifting in [26], we say that a digital lifting of $f$ is a $\left(k_{2}, k_{0}\right)$-continuous map $\tilde{f}: X \rightarrow E$ such that $p \circ \tilde{f}=f[6]$. Thus, the unique digital lifting theorem in [6] (see also [9, 12]) and digital homotopy lifting theorem was introduced in [5], which plays an important role in studying digital covering theory.

Although in algebraic topology it is well-known that a simply connected and locally path connected covering space is a universal covering space [28], in digital covering theory we can propose that a generalized universal covering space has its intrinsic feature. The following theorem has been often used in studying the digital lifting theorem.

Unlike the non-2-contractibility of $\mathbf{Z}$, we can observe the simply 2connectedness of $\mathbf{Z}$ [13], which can be essential to the proof of the generalized lifting theorem in $[3,13]$. While the universal property of a digital covering in [3] was studied for a radius $2-\left(k_{0}, k_{1}\right)$-covering with some hypothesis. As a generalization of the universal covering property of [3], we obtain the following. 
Definition 5. [16] We say that $(\tilde{E}, p, B)$ is a universal $(\tilde{k}, k)$-covering if for any radius 2 - $\left(k_{1}, k\right)$-covering map $q: \tilde{X} \rightarrow B$, there is always a $\left(\tilde{k}, k_{1}\right)$-continuous map $f:(\tilde{E}, \tilde{k}) \rightarrow\left(\tilde{X}, \tilde{k}_{1}\right)$ such that $q \circ f=p$.

In Definition 5 , the space $(\tilde{E}, \tilde{k})$ is called a universal $(\tilde{k}, k)$-covering space of $(B, k)$ and $(\tilde{E}, p, B)$ is called a universal $(\tilde{k}, k)$-covering. In addition, we say that $(\tilde{E}, \tilde{k})$ has the universal $(\tilde{k}, k)$-covering property. Indeed, the paper [3] studied the universal $(2, k)$-covering property.

As a generalization of the universal $(\tilde{k}, k)$-covering of Definition 5 , we can established the following:

Definition 6. [19] Let $\left(\left(E, e_{0}\right), \tilde{k}\right)$ and $\left(\left(B, b_{0}\right), k\right)$ be two digital spaces in $\mathbf{Z}^{n_{0}}$ and $\mathbf{Z}^{n_{1}}$, respectively. $A(\tilde{k}, k)$-covering map $p:\left(\left(E, e_{0}\right), \tilde{k}\right)$ $\rightarrow\left(\left(B, b_{0}\right), k\right)$ is called generalized universal if for any pointed $\left(k^{\prime}, k\right)$ covering map $q:\left(\left(X, x_{0}\right), k^{\prime}\right) \rightarrow\left(\left(B, b_{0}\right), k\right)$, there exists a pointed $\left(\tilde{k}, k^{\prime}\right)$-continuous map $\phi:\left(E, e_{0}\right) \rightarrow\left(X, x_{0}\right)$ such that $q \circ \phi=p$. Then, $\left(\left(E, e_{0}\right), k_{0}\right)$ is called a generalized universal $(\tilde{k}, k)$-covering space (briefly, $G U-(\tilde{k}, k)$-covering space) of $\left(\left(B, b_{0}\right), k\right)$. Furthermore, we say that this $(\tilde{k}, k)$-covering map $p$ has the generalized universal $(\tilde{k}, k)$ covering property (briefly, $G U$ - $(\tilde{k}, k)$-covering property). Besides, $\left(\left(E, e_{0}\right)\right.$, $\left.p,\left(B, b_{0}\right)\right)$ is called a generalized universal $(\tilde{k}, k)$-covering (briefly, $G U$ $(\tilde{k}, k)$-covering).

The current universal $\left(k_{0}, k_{1}\right)$-covering has no limitation of both the radius 2 - $\left(k_{0}, k_{1}\right)$-covering and the adjacency relations of $\left(E, k_{0}\right)$ in $\mathbf{Z}^{n_{0}}$ and $\left(B, k_{1}\right)$ in $\mathbf{Z}^{n_{1}}$ related to the $\left(k_{0}, k_{1}\right)$-covering $\left(\left(E, k_{0}\right), p,\left(B, k_{1}\right)\right)$, where $\left(\left(E, e_{0}\right), k_{0}\right)$ and $\left(\left(B, b_{0}\right), k_{1}\right)$ are two digital spaces in $\mathbf{Z}^{n_{0}}$ and $\mathbf{Z}^{n_{1}}$, respectively. In other words, in view of Definition 6 , if a given $\left(k_{0}, k_{1}\right)$ covering does not satisfy a radius 2 - $\left(k_{1}, k\right)$-isomorphism, then we cannot study further the universal $(\tilde{k}, k)$-covering property of $(\tilde{E}, p, B)$ in [3]. Thus, the paper [19] generalizes the universal $(2, k)$-covering without any limitation of $S C_{k}^{n, l}$ : Let $\left((\mathbf{Z}, 0), p,\left(S C_{k}^{n, l}, c_{0}\right)\right)$ be a $(2, k)$-covering. Then for any $\left(k_{0}, k\right)$-covering $\left(\left(X, x_{0}\right), q,\left(S C_{k}^{n, l}, c_{0}\right)\right)$, there is always a $\left(2, k_{0}\right)$-continuous map $f:(\mathbf{Z}, 0) \rightarrow\left(X, x_{0}\right)$ such that $q \circ f=p$. As an example of the GU- $(\tilde{k}, k)$-covering of Definition 6 , we obtained the following:

Theorem 3.3. [19] Consider a $\left(k^{\prime}, k\right)$-covering $\left(E, p, S C_{k}^{n, l}\right)$, where $S C_{k}^{n, l}$ need not be $k$-contractible. Then, $\left(E, p, S C_{k}^{n, l}\right)$ has the $G U-\left(k^{\prime}, k\right)$ covering property, where $\left(E, k^{\prime}\right)$ is $\left(k^{\prime}, 2\right)$-isomorphic to $(\mathbf{Z}, 2)$. 
Since the study of a digital covering space over a digital wedge is very important in digital covering theory, let us now recall a compatible adjacency of a digital wedge which is an advance form of the former in [6] (see also $[10,17]$ ).

Definition 7. [21] For pointed digital spaces $\left(\left(X, x_{0}\right), k_{0}\right)$ in $\mathbf{Z}^{n_{0}}$ and $\left(\left(Y, y_{0}\right), k_{1}\right)$ in $\mathbf{Z}^{n_{1}}$, the wedge of $\left(X, k_{0}\right)$ and $\left(Y, k_{1}\right)$, written $(X \vee$ $\left.Y,\left(x_{0}, y_{0}\right)\right)$, is the digital space in $\mathbf{Z}^{n}$

$$
\left\{(x, y) \in X \times Y \mid \quad x=x_{0} \quad \text { or } \quad y=y_{0}\right\}
$$

with the following compatible $k(m, n)$ (or $k$ )-adjacency relative to both $\left(X, k_{0}\right)$ and $\left(Y, k_{1}\right)$, and the only one point $\left(x_{0}, y_{0}\right)$ in common such that

(W1) the $k(m, n)$ (or $k$ )-adjacency is determined by the numbers $m$ and $n$ with $n=\max \left\{n_{0}, n_{1}\right\}, m=\max \left\{m_{0}, m_{1}\right\}$ satisfying $(W 1-1)$ below, where the numbers $m_{i}$ are taken from the $k_{i}\left(\right.$ or $\left.k\left(m_{i}, n_{i}\right)\right)$-adjacency relations of the given digital spaces $\left(\left(X, x_{0}\right), k_{0}\right)$ and $\left(\left(Y, y_{0}\right), k_{1}\right), i \in$ $\{0,1\}$.

(W 1-1) In view of (3.1), induced from the projection maps, we can consider the natural projection maps $W_{X}:\left(X \vee Y,\left(x_{0}, y_{0}\right)\right) \rightarrow\left(X, x_{0}\right)$ and $W_{Y}:\left(X \vee Y,\left(x_{0}, y_{0}\right)\right) \rightarrow\left(Y, y_{0}\right)$. In relation to the establishment of a compatible $k$-adjacency of the digital wedge $\left(X \vee Y,\left(x_{0}, y_{0}\right)\right)$, the following restriction maps of $W_{X}$ and $W_{Y}$ on $\left(X \times\left\{y_{0}\right\},\left(x_{0}, y_{0}\right)\right) \subset$ $\left(X \vee Y,\left(x_{0}, y_{0}\right)\right)$ and $\left(\left\{x_{0}\right\} \times Y,\left(x_{0}, y_{0}\right)\right) \subset\left(X \vee Y,\left(x_{0}, y_{0}\right)\right)$ satisfy the following properties, respectively:

$\left\{\begin{array}{l}\left.(1) W_{X}\right|_{X \times\left\{y_{0}\right\}}:\left(X \times\left\{y_{0}\right\}, k\right) \rightarrow\left(X, k_{0}\right) \text { is a }\left(k, k_{0}\right) \text {-isomorphism; and } \\ \left.(2) W_{Y}\right|_{\left\{x_{0}\right\} \times Y}:\left(\left\{x_{0}\right\} \times Y, k\right) \rightarrow\left(Y, k_{1}\right) \text { is a }\left(k, k_{1}\right) \text {-isomorphism. }\end{array}\right.$ (W2) Any two distinct elements $x\left(\neq x_{0}\right) \in X \subset X \vee Y$ and $y\left(\neq y_{0}\right) \in$ $Y \subset X \vee Y$ are not $k(m, n)$ (or $k$ )-adjacent to each other.

Example 3.4. [22] Consider the following three simple closed $k$ curves in $[4,8,15]$.

$M S C_{18}:=((0,0,0),(1,-1,0),(1,-1,1),(2,0,1),(1,1,1),(1,1,0)) \subset \mathbf{Z}^{3}$, $S C_{8}^{2,6} \approx_{8}((0,0),(1,1),(1,2),(0,3),(-1,2),(-1,1))$ and $S C_{26}^{3,4}:=((0,0,0),(1,1,1),(0,2,2),(-1,1,1))$.

Then we can consider digital wedges with compatible adjacency, as follows.

(1) $\left(M S C_{18} \vee S C_{8}^{2,6}, 18\right)$ and $\left(S C_{26}^{3,4} \vee S C_{8}^{2,6}, 26\right)$.

(2) No existence of compatible k-adjacency of $S C_{26}^{3,4} \vee M S C_{18}$.

Comparing with the former adjacency of a digital wedge in $[12,15]$, we obtain the following. 
Remark 3.5. The compatible adjacency of Definition 7 is a generalization of the former version in $[3,12,14]$.

\section{Some Properties of Infinite Fold Covering Spaces over a Digital Wedge Consisting of Two Simple Closed $k$-Curves}

In the study of an existence of an infinite fold covering space over $S C_{8}^{2,6} \vee S C_{8}^{2,6}$, the recent papers $[3,6,13,16,18,19]$ suggested two types of infinite fold covering spaces over $S C_{8}^{2,6} \vee S C_{8}^{2,6}$. Now we have the following question: How many kinds of infinite fold covering spaces over $S C_{k_{1}}^{n_{1}, l_{1}} \vee S C_{k_{2}}^{n_{2}, l_{2}}$ with a compatible $k$-adjacency in $\mathbf{Z}^{n}$. Thus, in this paper by using compatible adjacency of a digital wedge, we can study infinite fold digital covering spaces over a digital wedge consisting of two simple closed $k$-curves without any limitation of both a dimension and a digital wedge consisting of two simple closed $k$-curves.

Theorem 4.1. Assume $S C_{k_{1}}^{n_{1}, l_{1}} \vee S C_{k_{2}}^{n_{2}, l_{2}}$ with a compatible $k$-adjacency in $\mathbf{Z}^{n}$, where $n=\max \left\{n_{1}, n_{2}\right\}$. Then there are countably many infinite fold $\left(k^{\prime}, k\right)$-covering spaces $\left(E^{\prime}, k^{\prime}\right)$ in $\mathbf{Z}^{m}$ over $\left(S C_{k_{1}}^{n_{1}, l_{1}} \vee S C_{k_{2}}^{n_{2}, l_{2}}, k\right)$, $m \geq n$ such that

(1) each of them is the type 1 of Figure 1 and

(2) $\left(E^{\prime}, k^{\prime}\right)$ is $\left(k^{\prime}, 8\right)$-isomorphic to an infinite fold $(8,8)$-covering space in $\mathbf{Z}^{2}$ over $\left(S C_{8}^{2, l_{1}} \vee S C_{8}^{2, l_{2}}, 8\right)$.

Proof: If $S C_{k_{1}}^{n_{1}, l_{1}} \vee S C_{k_{2}}^{n_{2}, l_{2}}$ has a compatible $k$-adjacency in $\mathbf{Z}^{n}, n=$ $\max \left\{n_{1}, n_{2}\right\}$, then it is $(k, 8)$-isomorphic to $\left(S C_{8}^{2, l_{1}} \vee S C_{8}^{2, l_{2}}, 8\right)$ because each point $x \in S C_{k_{1}}^{n_{1}, l_{1}} \vee S C_{k_{2}}^{n_{2}, l_{2}}$, which is not the common point, has $N_{k}(x, 1) \subset S C_{k_{1}}^{n_{1}, l_{1}} \vee S C_{k_{2}}^{n_{2}, l_{2}}$ such that $N_{k}(x, 1) \approx_{(k, 2)} N_{2}(0,1)$ in $\mathbf{Z}$ and further, the common point denoted by $x_{0} \in S C_{k_{1}}^{n_{1}, l_{1}} \vee S C_{k_{2}}^{n_{2}, l_{2}}$ has $N_{k}\left(x_{0}, 1\right) \approx_{(k, 8)} N_{8}\left(v_{0}, 1\right)$, where $N_{8}\left(v_{0}, 1\right) \subset S C_{8}^{2, l_{1}} \vee S C_{8}^{2, l_{2}}$ and $v_{0}$ is the common point of the digital wedge of $S C_{8}^{2, l_{i}}, i \in\{1,2\}$. Therefore, it implies that there is a local $(k, 8)$-isomorphic bijection between $\left(S C_{k_{1}}^{n_{1}, l_{1}} \mathrm{~V}\right.$ $\left.S C_{k_{2}}^{n_{2}, l_{2}}, k\right)$ and $\left(S C_{8}^{2, l_{1}} \vee S C_{8}^{2, l_{2}}, 8\right)$. In view of this assertion, for some $m \in \mathbf{N}$ with $m \geq n$ (here the number $m$ need not be equal to $n$ ), we obtain an infinite fold $\left(k^{\prime}, 8\right)$-covering space $\left(E^{\prime}, k^{\prime}\right) \subset \mathbf{Z}^{m}$ over $\left(S C_{8}^{2, l_{1}} \vee\right.$ $\left.S C_{8}^{2, l_{2}}, 8\right)$ having the type 1 of Figure 1 , and further, $\left(E^{\prime}, k^{\prime}\right) \approx_{\left(k^{\prime}, 8\right)}$ $(F, 8)$, where $(F, 8)$ is an infinite fold $(8,8)$-covering space over $S C_{8}^{2, l_{1}} \vee$ $S C_{8}^{2, l_{2}}$ (see the covering space over $S C_{8}^{2, l_{1}} \vee S C_{8}^{2, l_{2}}$ in Figure 1(b)). As 
presented in (a) of Figure 1, since the object $\left(E^{\prime}, k^{\prime}\right)$ is assumed in $\mathbf{Z}^{m}$ with $m \geq n$ with $n=\max \left\{n_{1}, n_{2}\right\}$, the existence of $\left(E^{\prime}, k^{\prime}\right)$ in $\mathbf{Z}^{m}$ is valid. For instance, for any finite numbers $l_{i}$ of $S C_{k_{i}}^{n_{i}, l_{i}}, i \in\{0,1\}$ we can figure out the two digital spaces $\left(E^{\prime}, k^{\prime}\right)$ and $(F, 8)$ presented in (a) and (b) of Figure 1.

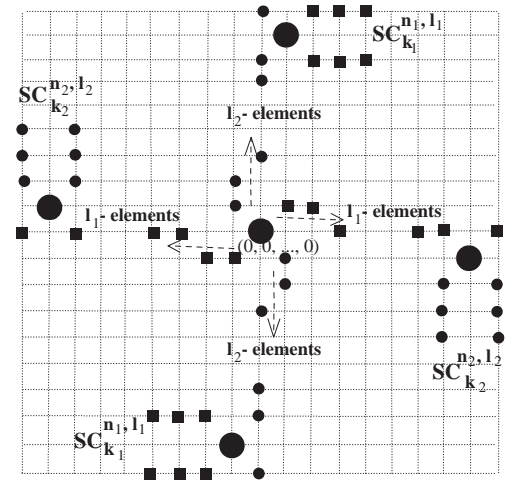

(Type 1) in $\mathbf{Z}^{\mathrm{m}} \quad \mathrm{E}^{\prime}$

(a) $\downarrow \mathbf{p}$ $\mathbf{S C}_{\mathbf{k}_{1}}^{\mathbf{n}_{1}, \mathbf{l}_{1}} \mathbf{V} \mathbf{S C}_{\mathbf{k}_{2}}^{\mathbf{n}_{2}, \mathbf{l}_{2}}$

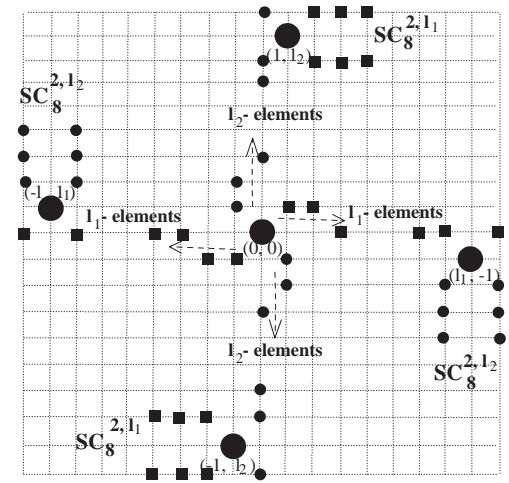

(Type 2) in $\mathrm{Z}^{2}$ $\mathrm{SC}_{8}^{2,1_{1}} \mathrm{~V} \mathrm{SC}_{8}^{2,1_{2}}$

Figure 1. (a) Infinite fold $\left(k^{\prime}, k\right)$-covering $\left(E^{\prime}, p, S C_{k_{1}}^{n_{1}, l_{1}} \vee S C_{k_{2}}^{n_{2}, l_{2}}\right)$ in $\mathbf{Z}^{m}, m \geq n, n=\max \left\{n_{1}, n_{2}\right\}$ (b) Infinite fold $(8,8)$-covering $\left(F, p, S C_{8}^{2, l_{1}} \vee S C_{8}^{2, l_{2}}\right)$.

Example 4.2. Consider the two digital wedges $\left(M S C_{18} \vee S C_{8}^{2,6}, 18\right)$ and $\left(S C_{26}^{3,4} \vee S C_{8}^{2,6}, 26\right)$ in Example 3.4. By using the same method as Theorem 3.1, we can find countably many infinite fold $\left(3^{n}-2^{n}-1,18\right)$ covering spaces $\left(E^{\prime}, p, M S C_{18} \vee S C_{8}^{2,6}, 18\right), n \geq 3$ such that $\left(E^{\prime}, 3^{n}-\right.$ $\left.2^{n}-1\right)$ is $\left(3^{n}-2^{n}-1,8\right)$-isomorphic to an infinite fold $(8,18)$-covering space $\left(F_{1}, 8\right)$ in $\mathbf{Z}^{2}$ over $M S C_{18} \vee S C_{8}^{2,6}$ having the type 1 of Figure 1. Besides, by the same method as above, we can find countably many infinite fold $\left(3^{m}-1,26\right)$-covering spaces over $\left(S C_{26}^{3,4} \vee S C_{8}^{2,6}, 26\right)$ having the type 1 of Figure 1. 


\section{Remark on a Generalized Universal Covering Space}

In algebraic topology it is well-known that the existence problem of a universal covering space [28]: A simply connected and locally path connected covering space is a universal covering space. For a digital wedge consisting of two simple closed 8-curves, the papers $[3,16]$ deals with a generalized universal covering space in $\mathbf{Z}^{2}$. But in this paper, by using a compatible adjacency of a digital wedge of Definition 7 , we can discuss an existence problem of a generalized universal covering space over a digital wedge consisting of two simple closed $k$-curves without any limitation of dimensions of both a digital covering space and a digital wedge of two simple closed $k$-curves.

Remark 5.1. As discussed in [16], in view of each of digital covering spaces over $S C_{k_{1}}^{n_{1}, l_{1}} \vee S C_{k_{2}}^{n_{2}, l_{2}}$ in Figure 1, we obtain that the digital covering space $\left(E, k^{\prime}\right) \subset \mathbf{Z}^{m}$ in Theorem 4.1 cannot be a generalized universal covering space.

In addition, we can observe that the simply $k$-connected of a base space $(B, k)$ need not guarantee the existence of a $\mathrm{GU}-\left(k^{\prime}, k\right)$-covering, as follows.

Theorem 5.2. Consider a $(k, 8)$-covering $\left(E, p, S C_{8}^{2,4} \vee S C_{8}^{2,4}\right)$ such that $E$ is simply $k$-connected. Then, $\left(E, p, S C_{8}^{2,4} \vee S C_{8}^{2,4}\right)$ need not have the $G U-(k, 8)$-covering property.

Before proving this theorem, we had better comment the proof of the assertion of [18], as follows. In Theorem 5.2, we have corrected by replacing the word "cannot" of [18] by "need not". In order to prove the former version of Theorem 5.2, the paper [18] used an infinite fold covering space over $S C_{8}^{2,4} \vee S C_{8}^{2,4}$ (see Figure 1 of [18]). However, the picture is ambiguous and is not clear. Thus, the present paper will use a finite and simple example instead.

Proof: Consider an $(8,8)$-covering $(E, p, B)$ such that $E$ is simply 8-connected, where $B=S C_{8}^{2,4} \vee S C_{8}^{2,4}$. Then, $(E, p, B)$ need not have the GU- $(8,8)$-covering property. In order to prove the assertion, we suffice to give the following counter example. Consider the identity map $1_{B}: B \rightarrow B$ so that $E=B$. Further, consider the $(8,8)$-covering map $r: F \rightarrow B$ in Figure 2. Then, it is clear that the given identity map $1_{B}: B \rightarrow B$ cannot have an $(8,8)$-continuous map $q: B \rightarrow F$ such that $r \circ q=1_{B}$. 


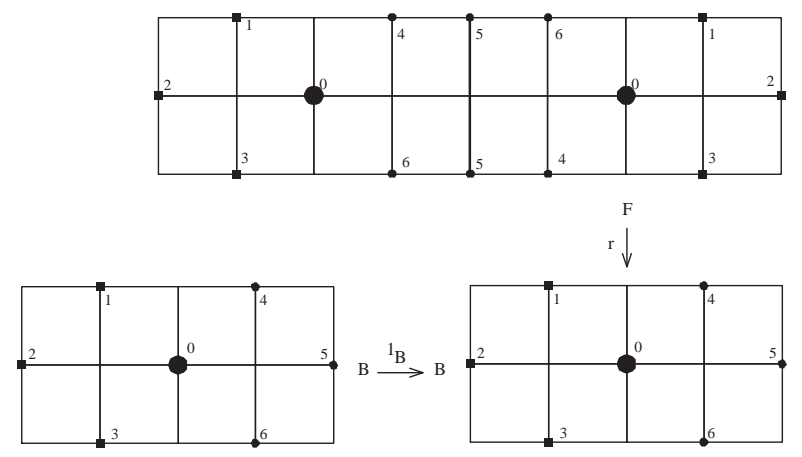

Figure 2. Configuration of a non-existence of the universal covering property.

In view of Theorem 5.2, we may have the following question.

[Question A] Does the digital wedge $\left(S C_{8}^{2,4} \vee S C_{8}^{2,4}, 8\right)$ have the universal covering space?

As a general form of Question A, we can suggest the following question. [Open question] Let $B:=S C_{k_{0}}^{n_{0}, l_{0}} \vee S C_{k_{1}}^{k_{1}, l_{1}}$ be a digital wedge with some compatible $k$-adjacency, where $S C_{k_{i}}^{n_{i}, l_{i}}$ need not be $k_{i}$-contractible, $i \in\{0,1\}$. Then we propose an open problem: under what condition of $B$ is there a universal $\left(k^{\prime}, k\right)$-covering space over $B$ ?

\section{References}

[1] R. Ayala, E. Domínguez, A.R. Francés, and A. Quintero, Homotopy in digital spaces, Discrete Applied Math, 125(1) (2003) 3-24.

[2] L. Boxer, A classical construction for the digital fundamental group, Jour. of Mathematical Imaging and Vision, 10(1999) 51-62.

[3] L. Boxer, Digital Products, Wedge; and Covering Spaces, Jour. of Mathematical Imaging and Vision 25(2006) 159-171.

[4] S.E. Han, Algorithm for discriminating digital images w.r.t. a digital $\left(k_{0}, k_{1}\right)$ homeomorphism, Jour. of Applied Mathematics and Computing 18(1-2)(2005) 505-512.

[5] S.E. Han, Digital coverings and their applications, Jour. of Applied Mathematics and Computing 18(1-2)(2005) 487-495.

[6] S.E. Han, Non-product property of the digital fundamental group, Information Sciences 171 (1-3)(2005) 73-91.

[7] S.E. Han, On the simplicial complex stemmed from a digital graph, Honam Mathematical Journal 27 (1)(2005) 115-129.

[8] S.E. Han, Erratum to "Non-product property of the digital fundamental group", Information Sciences 176(1)(2006) 215-216. 
[9] S.E. Han, Discrete Homotopy of a Closed $k$-Surface, LNCS 4040, SpringerVerlag, Berlin, pp.214-225 (2006).

[10] S.E. Han, Minimal simple closed 18-surfaces and a topological preservation of 3D surfaces, Information Sciences 176(2)(2006) 120-134.

[11] S.E. Han, Strong $k$-deformation retract and its applications, Journal of the Korean Mathematical Society 44(6)(2007) 1479-1503.

[12] S.E. Han, Comparison among digital fundamental groups and its applications, Information Sciences 178(2008) 2091-2104.

[13] S.E. Han, Equivalent $\left(k_{0}, k_{1}\right)$-covering and generalized digital lifting, Information Sciences 178(2)(2008)550-561.

[14] S.E. Han, Map preserving local properties of a digital image Acta Applicandae Mathematicae 104(2) (2008) 177-190.

[15] S.E. Han, The $k$-homotopic thinning and a torus-like digital image in $\mathbf{Z}^{n}$, Journal of Mathematical Imaging and Vision 31 (1)(2008) 1-16.

[16] S.E. Han, Cartesian product of the universal covering property Acta Applicandae Mathematicae 108 (2009) 363-383.

[17] S.E. Han, Regural covering space in digital covering theory and its applications, Honam Mathematical Journal 31(3) (2009) 279-292.

[18] S.E. Han, Remark on a generalized universal covering space, Honam Mathematical Jour 31(3) (2009) 267-278.

[19] S.E. Han, Existence problem of a generalized universal covering space, Acta Applicandae Mathematicae 109(3) (2010) 805-827.

[20] S.E. Han, KD- $\left(k_{0}, k_{1}\right)$-homotopy equivalence and its applications Journal of Korean Mathematical Society 47(5) (2010) 1031-1054.

[21] S.E. Han, Ultra regular covering space and its automorphism group International Journal of Applied Mathematics Computer Science, accepted.

[22] S.E. Han, Properties of a digital covering spaces and discrete Deck's transformation group Acta Applicandae Mathematicae, submitted

[23] E. Khalimsky, Motion, deformation, and homotopy in finite spaces, Proceedings IEEE International Conferences on Systems, Man, and Cybernetics(1987) 227234.

[24] T.Y. Kong, A digital fundamental group Computers and Graphics 13 (1989) 159-166.

[25] T.Y. Kong, A. Rosenfeld, Topological Algorithms for the Digital Image Processing, Elsevier Science, Amsterdam, (1996).

[26] W.S. Massey, Algebraic Topology, Springer-Verlag, New York, 1977.

[27] A. Rosenfeld, Digital topology, Am. Math. Mon. 86(1979) 76-87.

[28] E.H. Spanier, Algebraic Topology, McGraw-Hill Inc., New York, 1966. 
Properties of a Generalized Universal Covering Space over a Digital Wedge... 387

Faculty of Liberal Education,

Institute of Pure and Applied Mathematics,

Chonbuk National University,

Jeonju-City Jeonbuk, 561-756,

Republic of Korea

Tel: +82-63-270-4449,

E-mail:sehan@jbnu.ac.kr 